\title{
Long-Term Vasectomy
}

\author{
EFFECTS ON THE OCCURRENCE AND EXTENT \\ OF ATHEROSCLEROSIS IN RHESUS MONKEYS
}

\author{
Thomas B. Clakkson and Nancy J. Alexander, Arteriosclerosis Research Center, \\ Bowman Gray School of Medicine, Wake Forest University, \\ Winston-Salem, North Carolina 27103; Reproductive Physiology, \\ Oregon Regional Primate Research Center, Beaverton, Oregon 97005
}

A B S TRACT We demonstrated previously that atherosclerosis develops more extensively in vasectomized cynomolgus macaques fed an atherogenic diet and speculated that the immunologic response to sperm antigens may have exacerbated the atherosclerosis. We report here that rhesus monkeys vasectonized for 9-14 yr and fed monkey chow (devoid of cholesterol and low in fat) rather than an atherogenic diet also had more extensive and severe atherosclerosis than did control animals of the same age.

The extent of atherosclerosis was considered as the percentage of intimal surface with plaques. No control animals were found to have plaques in the thoracic aorta, but 7 of 10 vasectomized monkeys were affected. The plaques in the vasectomized monkeys occupied about $13 \%$ of the intimal surface. In 4 of 7 control monkeys and 7 of 10 vasectomized monkeys there were lesions in the abdominal aortas; the lesions were considerably more extensive and severe in the vasectomized animals. Lesions were also more common in iliac arteries of vasectomized animals, and the extent was increased about threefold.

Plaques were seen at the carotid bifurcation in all of the animals of both the control and vasectomized groups. The carotid bifurcation plaques of the vasectomized monkeys were larger than those of the control animals on the right but not on the left side.

Histologically, the lesions of vasectomized monkeys did not appear to be qualitatively different from those of control animals, even though they were larger and contained more collagen, lipid, and mucopolysaccharides. Grossly, the distribution of the lesions in the vasectomized animals was different from that in the control animals, and that of lesions induced by athero-

Received for publication 9 April 1979 and in revised form 13 August 1979. genic diets, i.e., the lesions were distributed randomly within the artery rather than around bifurcations.

More extensive atherosclerosis was noted among vasectomized animals that were found to lack demonstrable circulating free antisperm antibodies. On the basis of the observations made in this study, we suggest that the antisperm antibodies that form after vasectomy may result in circulating immune complexes that exacerbate atherosclerosis.

\section{INTRODUCTION}

During the past decade, the exacerbation of atherosclerosis by immunologic injury to arteries has become of considerable interest. The combination of serum sickness (immune complex disease) and hyperlipoproteinemia has been shown in rabbits to result in more extensive atherosclerosis than hyperlipoproteinemia alone (1). Furthermore, the results of other experiments with rabbits have shown that the combination of repeated immunologic injury to arteries and a lipidrich diet not only increases the extent of atherosclerosis, but also results in changes in the anatomical location and histological characteristics of the plaques so that they more nearly resemble the fibromuscular atherosclerotic plaques of human beings (2). Immunologic injury has also been shown to enhance the development of atherosclerosis in baboons (3). Repeated immunization with foreign protein plus being fed a lipid-rich diet resulted in much more severe atherosclerosis than being fed only the lipid-rich diet.

These studies on experimentally induced immunologic injury to arteries prompted us to investigate the effects of vasectomy on the pathogenesis of atherosclerosis. After vasectomy, antibodies to sperm develop in about $50 \%$ of vasectomized men $(4-7)$ as well as in vasectomized males of other species (8-10). Spermatozoa contain numerous antigens, both on their 
surface and internally. Vasectomy results in blockage of their normal anatomical passage allowing for possible degeneration in situ and release of soluble antigens that may enter the circulation directly. Sperm agglutination, serum immobilization, and immunofluorescence have all been used as means to demonstrate circulating antibodies to sperm in vasectomized individuals. The presence of such antibodies indicates antigen leakage and activation of the immune system.

In earlier studies we investigated the effects of vasectomy on the extent and severity of diet-induced atherosclerosis in Macaca fascicularis (11). We found that diet-induced atherosclerosis developed more extensively in vasectomized than in sham-vasectomized monkeys fed the same diet. Antibodies to sperm developed in all vasectomized monkeys, and complement and immunoglobulins were associated with the atherosclerotic plaques in some of the vasectomized animals. On the basis of those studies, we speculated that the immunologic response to vasectomy resulted in injury to the vascular endothelium and thereby resulted in a more rapid progression of the atherosclerosis.

In this report we describe the atherosclerotic lesions that developed in rhesus monkeys (Macaca mulatta) vasectomized for long periods and fed only fruit and commercial monkey chow (devoid of cholesterol and very low in fat).

\section{METHODS}

Animals. The monkeys were all housed at the Oregon Regional Primate Research Center (ORPRC). ${ }^{1}$ The animals used in these studies were adult male $M$. mulatta that had been vasectomized for periods of 9-14 $\mathrm{yr}$ and intact controls that were deemed to be of equivalent ages to the animals in the vasectomy group. There were 8 male control and 10 vasectomized monkeys; 2 had been vasectomized for 9 yr, 5 for 10 $\mathrm{yr}$ and 3 for $14 \mathrm{yr}$. All of the animals that were vasectomized and five of the eight control animals were acquired from their country of origin (India) as young adults (6-8 yr of age). Three of the control animals were born at ORPRC and thus, their exact age was known. The age of the animals acquired from India was, of course, an estimate; however, estimation of age of young adults on the basis of dentition is considered accurate within $1 \mathrm{yr}$. All of the animals had been in captivity and fed normal monkey chow for $10 \mathrm{yr}$ or more at least 7 of which had been at ORPRC.

Considering either their estimated age on arrival from India or the exact age of those born at ORPRC, it is possible to either estimate or know the age of the animals at necropsy. There were three animals vasectomized in 1964 that were 20-22 yr old at necropsy; two control animals were 20-22 yr of age at necropsy. There were five animals vasectomized in 1968 that were 16-18 yr of age at necropsy; three control animals were 15-16 yr of age at necropsy; the two animals vasectomized in 1971 were $13-15 \mathrm{yr}$ old at necropsy; three control animals were known to be $14 \mathrm{yr}$ old at necropsy.

${ }^{1}$ Abbreviations used in this paper: BGSM, Bowman Gray School of Medicine; ORPRC, Oregon Regional Primate Research Center.
All monkeys were weighed every $30 \mathrm{~d}$ and tested for tuberculosis every $60 \mathrm{~d}$. No differences in the body weights were observed between the groups.

Diet. The animals were fed twice daily a diet of Purina monkey chow (15\% protein; Ralston Purina Co., St. Louis, Mo.), devoid of cholesterol, that contained about $6 \%$ fat derived primarily from plant sources. The animals were given fruit supplementation (oranges, apples, and bananas) three times a week.

Clinicopathological and endocrinological determinations. During the course of the study of these animals, a considerable amount of clinicopathological data were acquired. Measurements made on serum included the concentrations of electrolytes, calcium, phosphorus, triglycerides, total cholesterol, urea nitrogen, creatinine, albumin, and globulin; and the activities of alkaline phosphatase, glutamic oxaloacetic transaminase, and lactic dehydrogenase. All of the determinations except the albumin and globulin measurements were made with a 20 channel special memory address counter and the appropriate Technicon procedures (Technicon Instruments Corp., Tarrytown, N. Y.). The plasma proteins were separated by electrophoresis. Circulating concentrations of testosterone, dihydrotestosterone, estrone, estradiol, folliclestimulating hormone, and luteinizing hormone were determined for both the control and long-term vasectomized animals at ORPRC.

At the termination of the study a sample of serum was obtained for determination of total serum cholesterol and triglyceride concentrations. These determinations were made using the AutoAnalyzer II (Technicon Instruments Corp.) method of Rush et al. (12). The determinations were done at the Lipid Analytic Laboratory of the Arteriosclerosis Research Center, Bowman Gray School of Medicine (BGSM). This Laboratory is in the surveillance stage of the quality control program of the Center for Disease Control, Atlanta, Georgia.

Immunologic determinations. Sperm-agglutination, sperm-immobilization, and immunofluorescence tests were used in the assessment of circulating antibody titers against sperm in all the animals $(6,13,14)$. These determinations were done twice per year for the last $7 \mathrm{yr}$ on the vasectomized group and for the last $4 \mathrm{yr}$ on the control group.

Necropsy procedure. Gross necropsies were done at the ORPRC. Each animal was anesthetized with ketamine and exsanguinated from the inferior vena cava. At the time of necropsy the carotid arteries, the thoracic aortas, the abdominal aortas, and the iliac arteries were opened longitudinally, and flattened on cardboard. Where lesions could be seen grossly, a 5-mm square sample was removed, placed on paper, and frozen in liquid nitrogen for evaluation with immunofluorescence. The remainder of the arteries were fixed in $10 \%$ neutral buffered formalin. Because it was not known then that considerable attention would be focused on the cardiovascular system, brains were not retained for evaluation of the cerebral circulation, and portions of the hearts of only a few animals were saved. The fixed arteries of the control and vasectomized monkeys were coded so that the group from which they came could not be determined and then shipped to the Arteriosclerosis Research Center, BGSM, for pathological evaluation.

Pathological evaluations. The arteries were stained overnight in a supersaturated solution of Sudan IV in $38 \%$ isopropanol for evaluation of the extent of grossly visible atherosclerosis. They were then washed in water and stored in $10 \%$ neutral buffered formalin. Each artery coded so that the evaluator (Dr. Clarkson) could not know whether it was from a control or vasectomized animal was studied separately. The percentage of intimal surface affected with raised 
atherosclerotic plaques was estimated visually and recorded. The gross evaluations were done three times to establish consistency of the grading method (average scores varied less than 5\%). The atherosclerotic involvement of the carotid bifurcation was considered separately, and severity was expressed on a relative scale of $0-4$.

For histological studies on the characteristics of the lesions, two adjacent or facing blocks were selected from each of the arteries from an area that represented the largest lesion seen and also from an area that represented the average lesion. For evaluation of the amount of stainable lipid in the lesions, one block was used for cryostat sectioning and was stained with Oil Red 0 and hematoxylin. The other block was processed in paraffin and $5-\mu \mathrm{m}$ sections were cut and stained with hematoxylin-phloxine-saffron or Verhoeff-van Gieson stains. Because of the need to quantify more accurately the carotid bifurcation atherosclerosis, we measured the maximal intimal thickness and plaque area with a pressure activated, hand-held digitizer interfaced with a computer.

Immunofuorescent evaluation. To locate possible immune complexes in frozen sections of arteries, we used fluorescein isothiocyanate-labeled antibodies to immunoglobulin (Ig)G and IgM and to the third component of complement (C3) (N. L. Cappel Laboratories, Inc., Cochranville, $\mathrm{Pa}$.). The specificity of the conjugates was checked by immunoelectrophoresis. Blocking experiments demonstrated the specificity of the response.

\section{RESULTS}

Clinicopathological determinations. The results of the clinicopathologic and endocrinologic determinations with two exceptions revealed no differences between the long-term vasectomized animals and their age-matched controls $(15,16)$. First, the gamma globulin levels of the long-term vasectomized monkeys were significantly higher than those of the age-matched controls and the magnitude of the difference was about the same for vasectomized monkeys with and without detectable free antisperm antibodies. The $\alpha 1-, \alpha 2-$, and $\beta$-globulin concentrations of the two groups did not differ, nor did the luteinizing hormone, follicle-stimulating hormone, estrone, estradiol, testosterone, or dihydrotestosterone concentrations $(15,16)$.

Second, there was a suggestion of somewhat higher $(\sim 20 \mathrm{mg} / \mathrm{dl})$ serum triglyceride concentrations among animals with high circulating levels of antisperm antibodies $(15,16)$. These observations were made $\sim 2 \mathrm{yr}$ before necropsy, were made using special memory address counter procedures, and whether all of the animals had been fasted for the same time periods before sample collection is not known. At the termination of the study additional blood samples were obtained for serum lipid determinations at BGSM. We found no significant differences in serum triglyceride concentrations between the vasectomized and control groups (35 \pm 4 [SE] $\mathrm{mg} / \mathrm{dl}$ vs. $56 \pm 11 \mathrm{mg} / \mathrm{dl}$, respectively). Additionally, we found no differences between the groups in mean total serum cholesterol concentration. Control animals had a mean total serum choles- terol concentration of $106 \pm 12(\mathrm{SE}) \mathrm{mg} / \mathrm{dl}$; whereas that of the vasectomized animals was $123 \pm 12 \mathrm{mg} / \mathrm{dl}$.

Immunologic determinations. The occurrence of antisperm antibodies was studied in all of the animals in the vasectomized and control groups with spermagglutination, sperm-immobilization, and indirect immunofluorescence techniques. No antisperm antibodies were found in the control animals. In Table I we have summarized our observations on the circulating antisperm antibody responses of the vasectomized monkeys and have compared them with the antibody status of the control animals. An animal was arbitrarily categorized as having a positive antisperm antibody response if its serum was positive by two different tests. On the basis of previous studies we know that vasectomized monkeys develop antibodies several weeks after the operation and that the number of animals retaining antisperm antibody titers drops off by 4-6 mo later and then remains steady with about onehalf the animals having free antisperm antibodies. Data from monkeys in these experiments, collected for the past $7 \mathrm{yr}$ are consistent with this concept.

Pathological evaluations. In Table II we have summarized the effect of long-term vasectomy on the occurrence of atherosclerosis among the arteries studied. Statistical significance was determined with the Fisher exact test. Although a few fatty streaks were seen in the common carotid arteries of both the vasectomized and control animals, no plaques were seen in either group. In contrast, considerable atherosclerosis occurred at the carotid bifurcation, and all of the animals in both groups were affected. No plaques were seen in the thoracic aortas of the control animals, whereas 7 of the 10 vasectomized animals had plaques ( $P$ $<0.006)$. The differences in frequency of atherosclerotic plaques in the abdominal aortas were not significant (i.e., 4 of 7 control animals were affected and 7 of 10 vasectomized animals were affected). Iliac arteries, like the thoracic aortas, were more often affected with atherosclerotic plaques in vasectomized animals (9 of 10) than in control animals ( 3 of 7$)$.

Having examined separately the question of whether vasectomy affects the frequency with which particular arteries are affected by atherosclerosis, we compared the extensiveness of the lesions and included in our determination only those animals with lesions. These data are summarized in Table III. Statistical significance of the differences between means and determined with the Student's $t$ test modified for unequal variance. On the right side, atherosclerosis of the carotid bifurcations in the vasectomized animals was significantly more extensive than that in control animals $(P<0.05)$; on the left side, atherosclerosis did not differ significantly between groups. The plaques affecting the thoracic aortas of the vasectomized monkeys involved about $13 \%$ of the intimal surface. There were no plaques in 


\section{TABLE I}

Circulating Antisperm Antibody Status of the Control and Vasectomized Rhesus Monkeys*

\begin{tabular}{|c|c|c|c|c|}
\hline Group & $\begin{array}{l}\text { General } \\
\text { antibody } \\
\text { response }\end{array}$ & $\begin{array}{c}\text { Sperm- } \\
\text { immobilizing } \\
\text { antibodies }\end{array}$ & $\begin{array}{c}\text { Sperm- } \\
\text { agglutinating } \\
\text { antibodies }\end{array}$ & $\begin{array}{l}\text { Immuno- } \\
\text { fluorescence }\end{array}$ \\
\hline \multicolumn{5}{|c|}{ Age-matched controls } \\
\hline 1207 & - & - & - & Ac \\
\hline 16.36 & - & - & - & - \\
\hline 1658 & - & - & - & - \\
\hline 5020 & - & - & - & Ac \\
\hline 5531 & - & - & - & - \\
\hline 5530 & - & - & - & - \\
\hline 1398 & - & - & - & - \\
\hline 1561 & - & - & - & - \\
\hline \multicolumn{5}{|c|}{ Long-term vasectomized } \\
\hline \multicolumn{5}{|c|}{ Vasectomized-1964 (14 yr) } \\
\hline 1062 & - & - & - & Ac \\
\hline 1065 & - & - & - & Ac \\
\hline 1073 & - & - & - & - \\
\hline \multicolumn{5}{|c|}{ Vasectomized-1968 (10 yr) } \\
\hline 3148 & - & - & - & - \\
\hline 3150 & + & + & + & Ac \\
\hline 3151 & + & + & - & $\mathrm{Ac}, \mathrm{Eq}$ \\
\hline 3152 & - & - & - & $\mathrm{Ac}, \mathrm{Eq}$ \\
\hline 3157 & - & - & - & - \\
\hline \multicolumn{5}{|c|}{ Vasectomized - $1971(9 \mathrm{yr})$} \\
\hline 5316 & + & + & + & Ac \\
\hline 5532 & + & + & + & $\mathrm{Ac}, \mathrm{Pa}$ \\
\hline
\end{tabular}

Ac, acrosomal region of the sperm; Eq, equatorial region of the sperm; $\mathrm{Pa}$, postacrosomal region of the sperm.

${ }^{*}$ This table is a composite of data gathered twice a year over the last $7 \mathrm{yr}$ for the vasectomy group and $4 \mathrm{yr}$ for the control monkeys. The sperm immobilization value was $\infty$ in each case (all sperm killed in test). Sperm-agglutinating titers were $3150=1: 320,5316=1: 20,5532=1: 640$. Immunofluorescence titers ranged from $1: 10$ to $1: 80$.

the thoracic aortas of the control animals. The largest difference in the extent of atherosclerosis between the two groups was found in the abdominal aorta. The control animals had plaques that occupied an average of $4.5 \%$ of the intimal surface; those of the long-term vasectomy group had a mean intimal surface involvement of $34.6 \%(P<0.001)$.

Being concerned that the gross grading of the

TABLE II

Effect of Long-term Vasectomy on the Frequency of Atherosclerosis among Rhesus Monkeys Fed Monkey Chow*

\begin{tabular}{|c|c|c|c|c|c|c|}
\hline \multirow[b]{2}{*}{ Group } & \multicolumn{2}{|c|}{$\begin{array}{c}\text { Carotid } \\
\text { bifurcation }\end{array}$} & \multirow{2}{*}{$\begin{array}{c}\text { Thoracic } \\
\text { aorta }\end{array}$} & \multirow{2}{*}{$\begin{array}{l}\text { Abdominal } \\
\text { aorta }\end{array}$} & \multicolumn{2}{|c|}{ Iliac arteries } \\
\hline & Left & Right & & & Left & Right \\
\hline & & & No. animal & plaques/No. & & \\
\hline \multirow{3}{*}{$\begin{array}{l}\text { Age-matched controls } \\
\text { Long-term vasectomized }\end{array}$} & $8 / 8$ & $8 / 8$ & $0 / 8$ & $4 / 7$ & $3 / 7$ & $1 / 7$ \\
\hline & $9 / 9$ & $9 / 9$ & $7 / 10$ & $7 / 10$ & $9 / 10$ & $8 / 10$ \\
\hline & NS & NS & $P<0.006$ & NS & $P<0.05$ & $P<0.01$ \\
\hline
\end{tabular}

* The abdominal aorta and iliac arteries were not available for evaluation of one control animal; the carotid arteries were not available for evaluation of one vasectomized animal. 
TABLE III

Effects of Long-term Vasectomy on the Extent of Atherosclerosis among Rhesus Monkeys Fed Monkey Chow*

\begin{tabular}{|c|c|c|c|c|c|c|}
\hline \multirow[b]{2}{*}{ Group } & \multicolumn{2}{|c|}{ Carotid bifurcation } & \multirow{2}{*}{$\begin{array}{c}\text { Thoracic } \\
\text { aorta }\end{array}$} & \multirow{2}{*}{$\begin{array}{l}\text { Abdominal } \\
\text { aorta }\end{array}$} & \multicolumn{2}{|c|}{ Iliac arteries } \\
\hline & Left & Right & & & Left & Right \\
\hline Age-matched controls & $2.2 \pm 0.37$ & $1.6 \pm 0.26$ & 0 & $4.5 \pm 0.25$ & $5.6 \pm 2.34$ & 5.0 \\
\hline Long-term vasectomized & $\begin{array}{c}2.8 \pm 0.42 \\
\text { NS }\end{array}$ & $\begin{array}{l}2.6 \pm 0.37 \\
P<0.05\end{array}$ & $\begin{array}{c}13.6 \pm 4.20 \\
-\end{array}$ & $\begin{array}{l}34.6 \pm 6.52 \\
P<0.001\end{array}$ & $\begin{array}{c}14.4 \pm 3.06 \\
P<0.05\end{array}$ & $\begin{array}{c}15.0 \pm 2.67 \\
P<0.05\end{array}$ \\
\hline
\end{tabular}

* Extent of carotid bifurcation arteriosclerosis was graded on a scale of 0-4. Extent of arteriosclerosis of other arteries is an estimate of $\%$ of intimal surface with plaques, only those animals with lesions are included in the means. The values are means $\pm S E$. There is no variance in the right iliac artery of age-matched controls because there was a single lesion only.

carotid bifurcation atherosclerosis on a scale of $0-4$ might not be sufficiently precise to characterize possible differences between the groups, we made additional histological measurements. These included a determination of the maximum intimal thickness of each animal's carotid bifurcation plaque and the area of the plaque, a calculation that took into account both thickness and length of the lesion. These data are summarized in Table IV. Consistent with the gross grades were our findings $(a)$ that on the right side the carotid bifurcation plaques of the vasectomized animals were thicker than those of control animals and $(b)$ that on the left side the thickness of the plaques did not differ between groups.

We sought to determine if there was any relationship between the extent of atherosclerosis among the vasectomized animals that either did or did not have circulating free antisperm antibodies. In Table $\mathrm{V}$ we have shown separately the extent of the atherosclerosis in various arteries of the vasectomized animals whose test results for the last seven years were either positive or negative for free antisperm antibodies. There was more extensive atherosclerosis in the group that did not have free circulating antisperm antibodies. The differences in the two groups were striking, particularly in the abdominal aorta and iliac arteries.
The plaques seen at the carotid bifurcation of the control and vasectomized animals were typical fibrous or "pearly" plaques. The central portions of the plaques were usually glistening white and stained positively with the Sudan IV around the periphery (Fig. 1). Histologically, the plaques at the carotid bifurcation were more or less typical atherosclerotic plaques. They usually contained intercellular and extracellular lipid in the base with fibromuscular caps of varying thicknesses. In Fig. 2 we illustrate the characteristics of the lesions seen at the carotid bifurcation. Fig. 2A and $\mathrm{B}$ represent the diversity in lesions seen in the control animals. Lesions ranged from ones that were primarily intimal thickenings due to proliferated smooth muscle cells with minimal lipid accumulation (Fig. 2B) to ones that had a lipid core in the base and a fibromuscular cap (Fig. 2A). The carotid bifurcation lesions of the vasectomized animals appeared qualitatively similar but larger. Considerable lipid in the base of the lesions associated with a rather thick fibromuscular cap was common (Fig. 2D and E). Among the animals vasectomized for longer periods (14 yr), the lesions contained much more collagen and virtually all lipid was extracellular (Fig. 2C).

Atherosclerotic plaques of the thoracic aorta were seen only among the vasectomized animals. Several

TABLE IV

Gross and Histologic Evaluation of the Extent of Carotid Bifurcation Atherosclerosis among Control and Long-term Vasectomized Rhesus Monkeys Fed Monkey Chow*

\begin{tabular}{|c|c|c|c|c|c|c|}
\hline \multirow[b]{2}{*}{ Group } & \multicolumn{2}{|c|}{ Gross grade } & \multicolumn{2}{|c|}{ Maximal intimal thickness $t$} & \multicolumn{2}{|c|}{ Plaque areat } \\
\hline & Left & Right & Left & Right & Left & Right \\
\hline & & & \multicolumn{2}{|c|}{$m m$} & \multicolumn{2}{|c|}{$m m^{2}$} \\
\hline Age-matched controls & $2.2 \pm 0.37$ & $1.6 \pm 0.26$ & $0.26 \pm 0.04$ & $0.19 \pm 0.32$ & $0.65 \pm 0.11$ & $0.53 \pm 0.14$ \\
\hline Long-term vasectomized & $\begin{array}{c}2.8 \pm 0.42 \\
\text { NS }\end{array}$ & $\begin{array}{l}2.6 \pm 0.37 \\
P<0.05\end{array}$ & $\begin{array}{c}0.28 \pm 0.06 \\
\text { NS }\end{array}$ & $\begin{array}{c}0.39 \pm 0.08 \\
P<0.05\end{array}$ & $\begin{array}{c}1.17 \pm 0.32 \\
\text { NS }\end{array}$ & $\begin{array}{c}1.25 \pm 0.26 \\
P<0.05\end{array}$ \\
\hline
\end{tabular}

* Gross extent of carotid bifurcation arteriosclerosis was graded on a scale of 0-4.

‡ Determined with a hand-held digitizer interfaced with a computer. 
TABLE V

Relationship of the Presence or Absence of Free Antisperm Antibody to the Extent of Atherosclerosis

\begin{tabular}{cccccc}
\hline $\begin{array}{c}\text { Free } \\
\text { antibody } \\
\text { status* }\end{array}$ & $\begin{array}{c}\text { Monkey } \\
\text { No. }\end{array}$ & $\begin{array}{c}\text { Carotid } \\
\text { bifurcation } \ddagger\end{array}$ & $\begin{array}{c}\text { Thoracic } \\
\text { aorta } \$\end{array}$ & $\begin{array}{c}\text { Abdominal } \\
\text { aorta } \$\end{array}$ & $\begin{array}{c}\text { Iliac } \\
\text { arteries" }\end{array}$ \\
\hline Positive & 3150 & 1.0 & 5 & 0 & 10.0 \\
& 3151 & 3.5 & 5 & 0 & 5.0 \\
& 5316 & 1.0 & 0 & 0 & 0 \\
& 5532 & 2.0 & 0 & 2 & 5.0 \\
Negative & 1062 & 3.0 & 15 & 35 & 25.0 \\
& 1065 & - & 35 & 40 & 20.0 \\
& 1073 & 4.0 & 20 & 35 & 20.0 \\
& 3148 & 3.5 & 10 & 60 & 17.5 \\
& 3152 & 4.0 & 0 & 30 & 10.0 \\
& 3157 & 3.0 & 5 & 40 & 12.5 \\
\hline
\end{tabular}

* Data from Table I.

$\ddagger$ Carotid bifurcation, extent of carotid bifurcation atherosclerosis was graded on a scale of $0-4$, the value presented for each animal is the mean of the left and right score.

$\S$ Extent of atherosclerosis is expressed as the percentage of intimal surface with plaques.

"The extent of iliac artery atherosclerosis is expressed as the percentage of intimal surface with plaques. The value presented for each animal is the mean of the left and right score.

features of those lesions were seen consistently. At the base of the plaques, just superficial to the internal elastic lamina, were numerous smooth muscle cells with a longitudinal orientation. Both intracellular lipid and extracellular lipid were seen in the cryostat sections stained with Sudan IV. The accumulation of collagen was the most striking feature, and abundant glycosaminoglycans were seen in sections stained with periodic acid-Schiff.

The appearance of the plaques in the abdominal aortas of the vasectomized animals was similar to that of typical fibrous or pearly plaques (Fig. 3). The distribution of the lesions was different from that seen in rhesus monkeys with diet-induced atherosclerosis. It also differed from the distribution of naturally occurring lesions of control monkeys in that the plaques were not associated with bifurcations or other areas of hemodynamic disturbance but rather appeared randomly in the artery. Histologically, the plaques that occurred in the abdominal aortas of the control animals were typical atherosclerotic plaques, with cores of lipid in the bases of the lesions and distinct fibromuscular caps. The abdominal aortic plaques of the vasectomized animals were larger and thicker, and had much more collagen associated with the fibromuscular caps.

The lesions in the iliac arteries of the control animals were fibrous in appearance and occurred most often at points of bifurcation. They were composed primarily

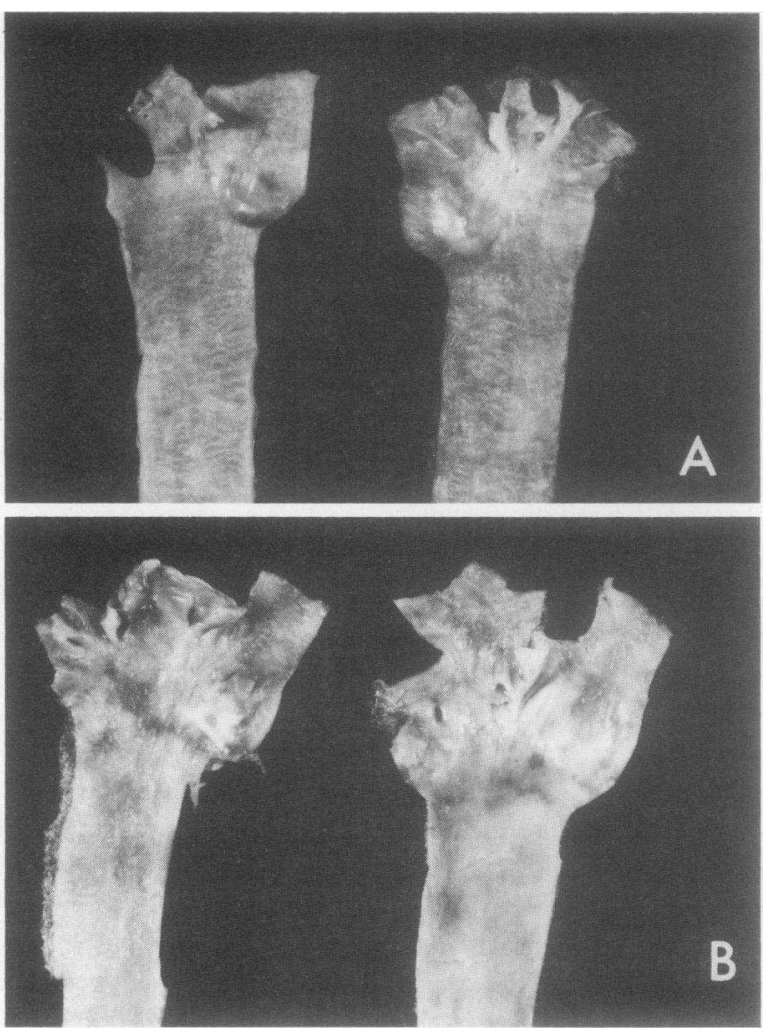

FIGURE 1 Photographs of right and left carotid bifurcations. $A$ is typical of control animals, B is typical of the vasectomized group. In both $A$ and $B$, glistening raised plaques can be seen at the bifurcation with Sudanophilia surrounding the plaque area.

of smooth muscle cells, collagen, and elastic fibers (Fig. 4). In contrast, the plaques seen in the iliac arteries of the vasectomized monkeys were not at bifurcation points, were larger, and stained grossly with Sudan around the periphery of the lesion. Histologically, the iliac artery plaques contained both intracellular and extracellular lipid and thick fibromuscular caps (Fig. 5).

Immunoglobulins and occasionally complement were found associated with lesions. Since their pattern of deposition was similar to that of fibrin, it is unlikely that they were immune complexes.

\section{DISCUSSION}

In this report we have presented evidence that atherosclerosis is more frequent and more extensive among vasectomized monkeys than age-matched controls. The gross and microscopic appearance of the lesions is similar to lesions reported by others as resulting from continuing mechanical injury or by immunological injury, and we speculate that the arterial lesions in these vasectomized monkeys may have been a result of their immunologic response to sperm antigens. 

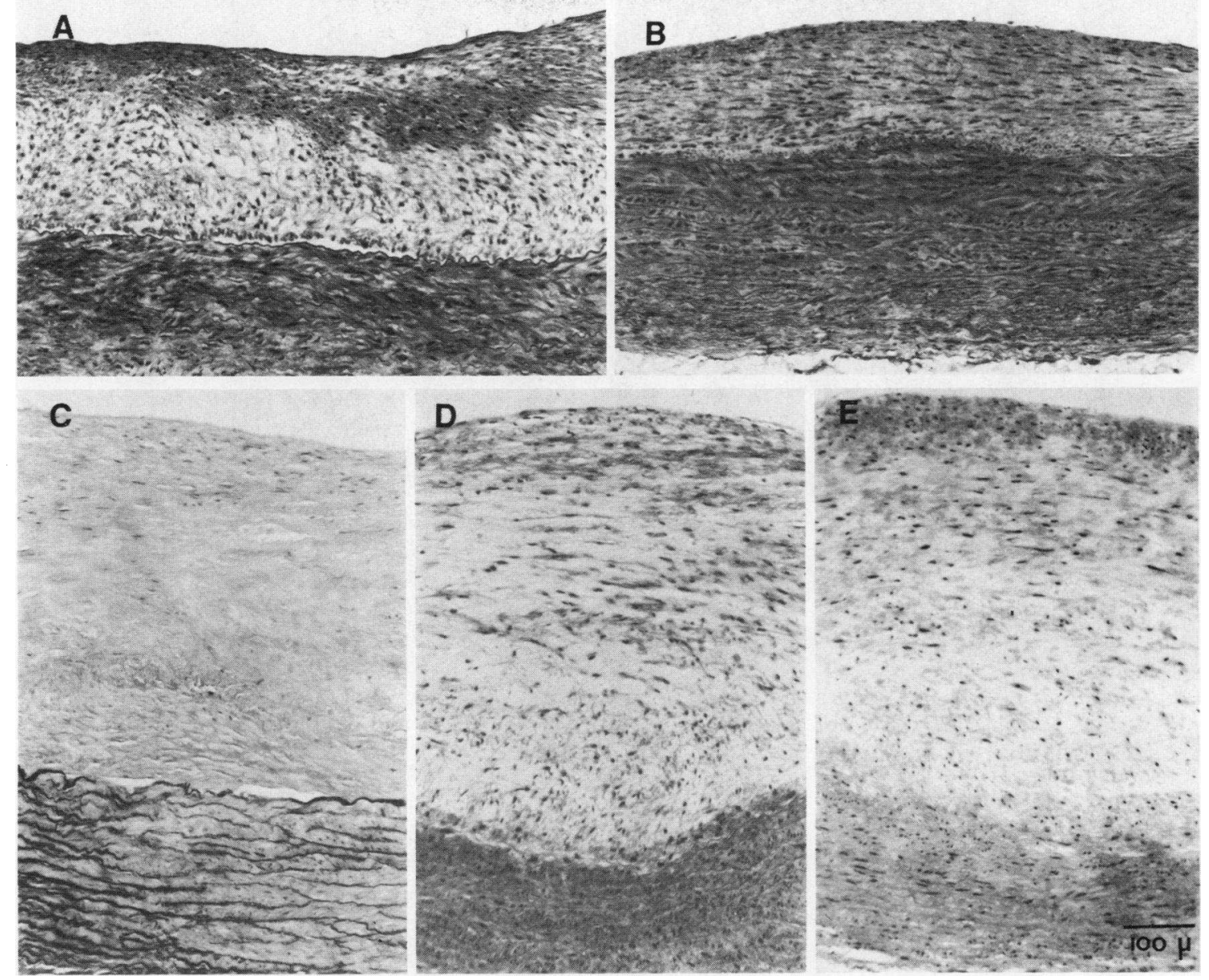

FIGURE 2 A and B are photomicrographs of sections of the carotid bifurcation of control animals. There is lipid in the deep part of the intima of A. The thickened intima of B contains mostly smooth muscle cells and their products. C, D, and E are sections from vasectomized animals. The intima of $C$ is collagenous and contains little lipid. There is increased lipid (clear areas in these paraffin sections), particularly extracellular lipid in D and E. A, B, D, and E were stained with hematoxylin-phloxine and saffron. $C$ was stained by the Verhoeff-van Gieson method. $(\times 100)$

The amount of naturally occurring atherosclerosis seen in the control animals was unexpected. Although the literature contains very little information on naturally occurring atherosclerosis in rhesus monkeys, the information that is available suggests that they are quite resistant to the development of naturally occurring lesions. Fox (17), on the basis of observations at the Philadelphia Zoo, concluded that rhesus monkeys were extremely resistant to naturally occurring atherosclerosis. Yakovleva (18) has reported on the occurrence of atherosclerosis in a single rhesus monkey that died at the estimated age of $27 \mathrm{yr}$. Chawla et al. (19) reported

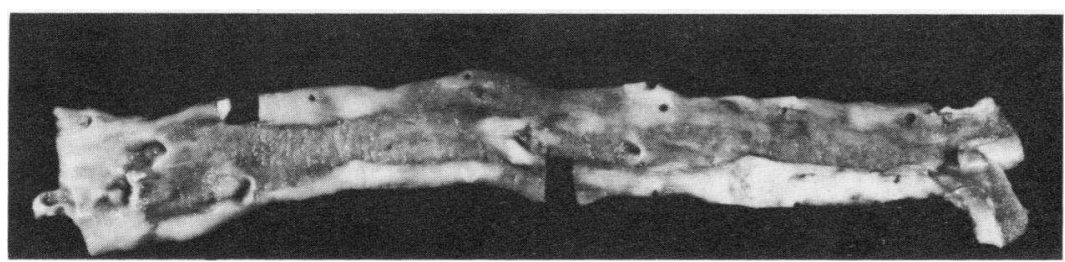

FIGURE 3 The abdominal aorta from a vasectomized monkey has extensive white, raised fibrous plaques. Stained grossly with Sudan IV. 


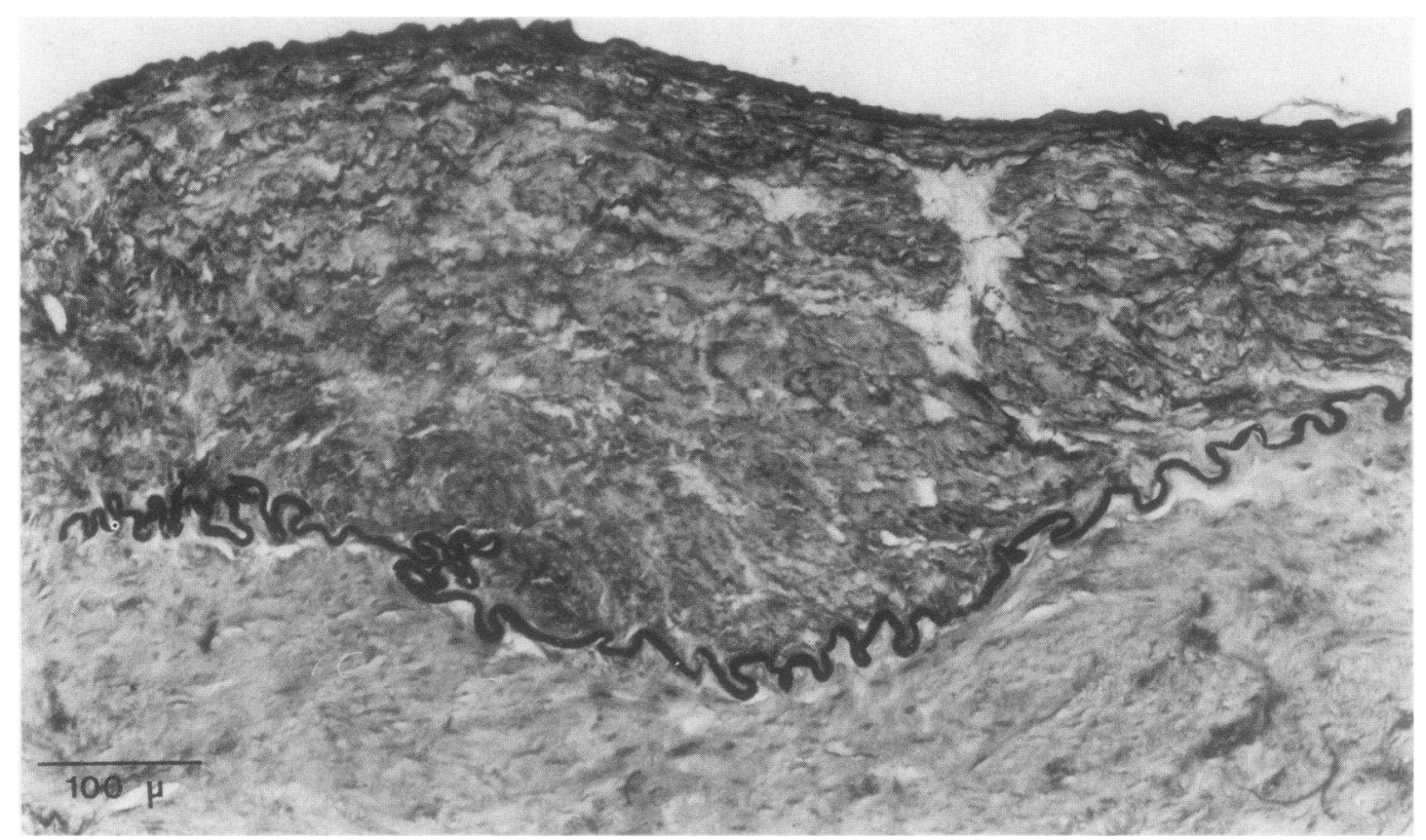

FIGURE 4 A plaque in the left iliac artery from a control monkey consists primarily of smooth muscle cells, collagen, and reduplicated elastic fibers. Stained by the Verhoeff-van Gieson method. $(\times 100)$

that $3 \%$ of 150 free-ranging rhesus monkeys had aortic fatty streaks; they saw no plaques. We have found no publications that dealt specifically with the carotid bifurcation, the site at which we saw the most advanced naturally occurring atherosclerosis in the control animals.

It is of interest that the carotid bifurcation atherosclerosis increased more on the right than the left side among the vasectomized animals. In a study by Solberg et al. (20) the percentage of intimal surface of carotid arteries with raised atherosclerotic lesions was somewhat greater for the right than the left carotid arteries of Guatemalan persons $65 \mathrm{yr}$ of age and older. Such differences were not seen in persons from Oslo.

The data presented seem to support the concept that exacerbation of atherosclerosis of the long-term vasectomized animals may have resulted from continuing immunologic injury to the arteries. Persistent antibody production such as is found in about $50 \%$ of men and animals after vasectomy suggests that a constant antigen source is present. Leakage of soluble sperm antigens would allow antigenemia and the development of circulating immune complexes. There are several pieces of evidence that point to the occurrence of circulating immune complexes as a result of vasectomy. First, vasectomized rabbits have glomerular changes which include an increased amount of mesangial matrix and granular deposition as seen by immunofluorescence. Eluates from such kidneys were shown to have antisperm antibodies (21). Second, circulating immune complexes have been found through the use of the Clq assay in the blood of vasectomized rabbits that had been injected with complete Freund's adjuvant (22) and orchiectomy of those vasectomized rabbits led to a precipitous fall in circulating immune complex levels and a rise in antibody levels. Rabbits with no antisperm antibody titers before castration had titers afterward. These observations provide strong evidence that the preexisting circulating immune complexes involved sperm antigens and antisperm antibodies. Removal of the antigen source made it possible to detect circulating free antibodies, because tests for antisperm antibody levels reveal free but not complexed antibody and are not measures of total antibody levels. The fact that castration resulted in antisperm antibodies shows that the antigens involved originated from the testis or epididymis.

Circulating immune complexes often result in arteritis. Studies in animals have shown that injection of any of several foreign proteins as well as the injection of preformed immune complexes can result in arteritis $(23,24)$.

Adherence of complexes to the endothelium allows the development of the complement cascade and the elaboration of chemotactic factors (particularly C5, C6, C7, C3a, and C5a). Polymorphonuclear leukocytes are attracted to the site and release their lysosomal enzymes, which diminish the integrity of the vascular endothelium resulting in increased permeability (25). 


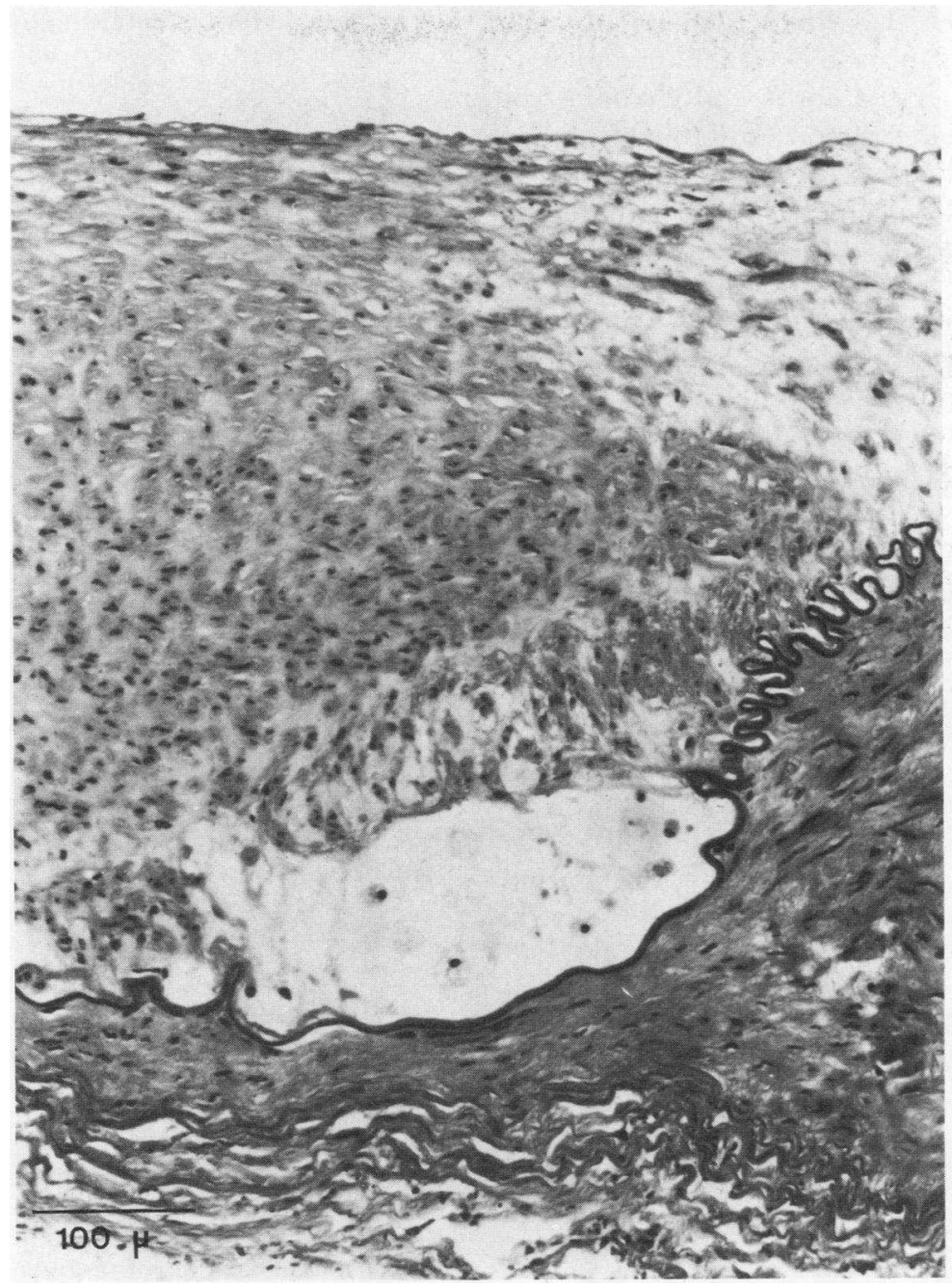

FIGURE 5 A photomicrograph of a section of a plaque in the right iliac artery of a vasectomized monkey. There is considerable lipid in the base of the plaque and in portions of the fibromuscular cap (clear areas in this paraffin section) and in portions of the thick fibromuscular cap. Stained with hematoxylin-phloxine and saffron. $(\times 100)$

Platelet accumulation and an inflammatory reaction also occur. Cellular debris is eventually removed by mononuclear cells.

Continued injury even without a diet containing cholesterol can result in fatty streaks and raised lipidcontaining lesions (26-28). Platelet factors are involved in continuing atherogenesis after injury (29). The pathologic characteristics of the arterial lesions that we observed in long-term vasectomized monkeys seem similar to those seen by Spaet et al. (30) in their studies of rabbit aortas that have been exposed to mechanical injury.

This sequence of events, i.e., circulating immune complexes, initiation of the complement cascade, influx of polymorphonuclear leukocytes, result in rapid degradation of immune complexes. In an Arthus reaction, the antigen is removed by polymorphonuclear leukocytes within $24 \mathrm{~h}$ (25). Thus, we were not surprised that we could not localize such complexes in the plaques of the long-term vasectomized monkeys.

In our previous study on the effects of vasectomy on diet-induced atherosclerosis of M. fascicularis (11), both the shamvasectomized and vasectomized groups of animals were markedly hypercholesterolemic (total plasma cholesterol concentrations were $500-600 \mathrm{mg} / \mathrm{dl}$ ). The immunologic injury associated with vasectomy exacerbated the atherosclerosis of those hyperlipoproteinemic animals, a finding consistent with the observations of other workers who used rabbits $(1,2)$ and baboons (3).

Earlier studies, on arterial injury in pigtail macaques (Macaca nemestrina), by Stemerman and Ross (31), 
provided some evidence, that immunologic injury may not exacerbate atherosclerosis in normolipoproteinemic animals. In their studies the aortic endothelium was damaged with a balloon catheter. Subsequently, they observed intimal thickenings of proliferated smooth muscle cells (without stainable lipid) that regressed from about 15 layers of smooth muscle cells to 2 or 3 cell layers within $6 \mathrm{mo}$ in normolipoproteinemic animals. When they induced injury in the same way in M. nemestrina fed dietary cholesterol (plasma cholesterol concentrations of $250-400 \mathrm{mg} / \mathrm{dl}$ ), the lesions did not regress and did accumulate stainable lipid. It seems likely that the difference in the mechanical injury that they studied and the presumed immunologic injury that we have studied is that in their model only a single episode of injury was used and in our animals there may well have been continuing immune-complex damage to the vascular endothelium over the 9- to 14-yr post-vasectomy span.

Of particular interest is our finding of more extensive atherosclerosis among vasectomized animals that had very probably developed antisperm antibodies initially but later lacked demonstrable circulating free antisperm antibodies. As we have indicated, almost all vasectomized rhesus monkeys develop antisperm antibodies shortly after vasectomy but by 4-6 mo only about one-half of the animals retain demonstrable free antisperm antibodies (32). One of the reasonable explanations for the lack of free antisperm antibodies is that such animals are in sperm antigen excess. On this basis, it seems reasonable to suggest, although we lack supporting data, that the animals in this experiment that lacked free antisperm antibody were in antigen excess and had greater concentrations of immune complexes.

\section{ACKNOWLEDGMENTS}

The authors thank Doctors B. C. Bullock and M. G. Bond, Bowman Gray School of Medicine for their helpful suggestions in the pathologic evaluations. We should also like to gratefully acknowledge the technical contributions of Kathryn Hubbard, Hermina Trillo, and Carol Marzetta and the contributions of Shirley Pegram in preparation of the manuscript. We thank Dr. J. Palotay, H. Hawash, D. F. Fulgham, and B. A. Mixon, Oregon Regional Primate Research Center for their assistance on the necropsies and later tissue preparations.

The work described in this article, Publication No. 1053 of the Oregon Regional Primate Research Center, was supported by National Institutes of Health HL 14164 at the Bowman Gray School of Medicine and contract N01-HD-4-2866 at the Oregon Regional Primate Research Center.

\section{REFERENCES}

1. Lamberson, H. V., Jr., and K. E. Fritz. 1974. Immunological enhancement of atherogenesis in rabbits. Persistent susceptibility to atherogenic diet following experimentally induced serum sickness. Arch. Pathol. 98: 9-16.

2. Minick, C. R., G. E. Murphy, and W. G. Campbell, Jr. 1966. Experimental induction of athero-arteriosclerosis by the synergy of allergic injury to arteries and lipid-rich diet. I. Effect of repeated injections of horse serum in rabbits fed a diet cholestrol supplement. J. Exp. Med. 124: 635-652.

3. Howard, A. N., J. Patelski, D. E. Bowyer, and G. A. Gresham. 1971. Atherosclerosis induced in hypercholesterolaemic baboons by immunological injury; and the effects of intravenous polyunsaturated phosphatidyl choline. Atherosclerosis. 14: 17-29.

4. Ansbacher, R., K. Keung-Yeung, and J. C. Wurster. 1972. Sperm antibodies in vasectomized men. Fertil. Steril. 23: 640-643.

5. Alexander, N. J., B. J. Wilson and G. D. Patterson. 1974. Vasectomy: Immunological effects in rhesus monkeys and men. Fertil. Steril. 25: 149-156.

6. Tung, K. S. K. 1975. Human sperm antigens and antisperm antibodies. I. Study on vasectomy patients. Clin. Exp. Immunol. 20: 93-104.

7. Samuel, T., A. H. J. Kolk, P. Rumke, and J. M. J. Van Lis. 1975. Autoimmunity to sperm antigens in vasectomized men. Clin. Exp. Immunol. 21: 65-74.

8. Rumke, P. H., and M. Titus. 1970. Spermagglutinin formation in male rats by subcutaneously injected syngeneic epididymal spermatozoa and by vasoligation or vasectomy. J. Reprod. Fertil. 21: 69-79.

9. Bigazzi, P. E., L. L. Kosuda, L. L. Harnick, R. C. Brown, and N. R. Rose. 1976. Antibodies to testicular antigens in vasectomized rabbits. Clin. Immunol. Immunopathol. 5: $182-194$.

10. Alexander, N. J., and K. S. K. Tung. 1977. Immunological and morphological effects of vasectomy in the rabbit. Anat. Rec. 188: 339-350.

11. Alexander, N. J., and T. B. Clarkson. 1978. Vasectomy increases the severity of diet-induced atherosclerosis in Macaca fascicularis. Science (Wash. D. C.). 201: 538-541.

12. Rush, R. L., L. Leon, and J. Turrell. 1971. Automated simultaneous cholesterol and triglyceride determination on the AutoAnalyzer II. In Advances in Automated Analysis-Technicon International Congress. E. C. Barton, editor. Thurman Associates, Miami, Fla. 1: 503-507.

13. Kibrick, S., D. L. Belding, and B. Merrill. 1952. Methods for the detection of antibodies against mammalian spermatozoa. II. A gelatin agglutination test. Fertil. Steril. 3: 430-438.

14. Isojiama, S., T. S. Li, and Y. Ashitaka. 1968. Immunologic analysis of sperm-immobilizing factor found in sera of women with unexplained sterility. Am. J. Obstet. Gynecol. 101: 677-683.

15. Alexander, N. J. 1975. Immunologic effects of vasectomy in rhesus monkeys. In Control of Male Fertility. J. J. Sciarra, C. Markland and J. J. Speidel, editors. Harper \& Row, Publishers, Inc., New York. 177-188.

16. Alexander, N. J., and K. S. K. Tung. 1979. Effects of vasectomy in rhesus monkeys. In Vasectomy: Immunologic and Pathophysiologic Effects. I. H. Lepow and R. Crozier, editors. Academic Press, Inc., New York. $423-458$.

17. Fox, H. 1933. Arteriosclerosis in Lower Mammals and Birds: Its Relation to the Disease in Man. In Arteriosclerosis: A Survey of the Problem. E. V. Cowdry, editor. MacMillan, Inc., New York.

18. Yakovleva, L. A. 1956. Theoretical and Practical Problems of Medicine and Biology in Experimentation on Monkeys. I. A. Utkin, editor. Medgiz, Moscow.

19. Chawla, K. K., C. D. S. Murthy, R. N. Chakravarti, and P. N. Chhuttani. 1967. Arteriosclerosis and thrombosis in wild rhesus monkeys. Am. Heart J. 73: 85-91.

20. Solberg, L. A., P. A. McGarry, J. Moossy, J. P. Strong, 
C. Tejada, and A. C. Loken. 1968. Severity of atherosclerosis in cerebral arteries, coronary arteries, and aortas. Ann. N. Y. Acad. Sci. 149: 956-973.

21. Bigazzi, P. E., L. L. Kosuda, K. C. Hsu, and G. A. Andres. 1976. Immune complex orchitis in vasectomized rabbits. J. Exp. Med. 143: 382-404.

22. Tung, K. S. K., R. K. Bryson, L.-P. B. Han, and L. C. Walker. 1979. Circulating immune complexes in vasectomy. In Vasectomy: Immunological and Pathophysiologic Effects. I. H. Lepow and R. Crozier, editors. Academic Press, Inc., New York.

23. Dixon, F. J., J. J. Vazquez, W. O. Weigle, and C. G. Cochrane. 1958. Pathogenesis of serum sickness. Arch. Pathol. 65: 18-28.

24. Germuth, F. G., and R. H. Heptinstall. 1957. The development of arterial lesions following prolonged sensitization of bovine gammaglobulin. Bull. Johns Hopkins Hosp. 100(No. 2): 58-70.

25. Cochrane, C. G., D. Hawkins, and W. T. Kniker. 1967. Mechanisms involved in the localization of circulating immune complexes in blood vessels. In Immunopathology. V. P. Miescher and P. Grabar, editors. Schwabe \& Co., Basel, Switzerland. 32.

26. Moore, S. 1973. Thrombo-atherosclerosis in normolipemic rabbits. A result of continued endothelial damage. $L a b$. Invest. 29: 478-487.

27. Friedman, R. J., S. Moore, and D. P. Singal. 1975. Repeated endothelial injury and induction of atherosclerosis in normolipemic rabbits by human serum. Lab. Invest. 30: 404-415.

28. Friedman, R. J., S. Moore, D. P. Singal, and M. Gent. 1976. Regression of injury induced atheromatous lesions in rabbits. Arch. Pathol. 100: 189-195.

29. Moore, S., R. J. Friedman, D. P. Singal, J. Gauldie, M. Blajchman, and R. Roberts. 1976. Inhibition of injury induced thromboatherosclerotic lesions by antiplatelet serum in rabbits. Thromb. Haemostasis. 35: 70-81.

30. Spaet, T. H., M. B. Stemerman, R. J. Friedman, and E. R. Burns. 1976. Arteriosclerosis in the rabbit aorta: Long-term response to a single ballon injury. Ann. N. Y. Acad. Sci. 275: 76-77.

31. Stemerman, M. D., and R. Ross. 1972. Experimental atherosclerosis. I. Fibrous plaque formation in primates, an electron microscope study. J. Exp. Med. 136: 769-789.

32. Alexander, N. J. 1975. Immunologic and morphologic effects of vasectomy in the rhesus monkey. Fed. Proc. 34:1692-1697. 